\title{
Improved removal of blood contamination from ThinPrep cervical cytology samples for Raman spectroscopic analysis
}

Damien Traynor

Shiyamala Duraipandian

Cara M. Martin

John J. O'Leary

Fiona M. Lyng 


\title{
Improved removal of blood contamination from ThinPrep cervical cytology samples for Raman spectroscopic analysis
}

\author{
Damien Traynor, ${ }^{\mathrm{a}, \star}$ Shiyamala Duraipandian, ${ }^{\mathrm{a}}$ Cara M. Martin, ${ }^{\mathrm{b}}$ John J. O’Leary, ${ }^{\mathrm{b}}$ and Fiona M. Lyng ${ }^{\mathrm{a}}$ \\ ${ }^{a}$ Dublin Institute of Technology (DIT), Focas Research Institute, DIT Centre for Radiation and Environmental Science, Dublin, Ireland \\ ${ }^{\mathrm{b} C}$ Coombe Women and Infants University Hospital, Department of Pathology, Dublin, Ireland
}

\begin{abstract}
There is an unmet need for methods to help in the early detection of cervical precancer. Optical spectroscopy-based techniques, such as Raman spectroscopy, have shown great potential for diagnosis of different cancers, including cervical cancer. However, relatively few studies have been carried out on liquid-based cytology (LBC) pap test specimens and confounding factors, such as blood contamination, have been identified. Previous work reported a method to remove blood contamination before Raman spectroscopy by pretreatment of the slides with hydrogen peroxide. The aim of the present study was to extend this work to excessively bloody samples to see if these could be rendered suitable for Raman spectroscopy. LBC ThinPrep specimens were treated by adding hydrogen peroxide directly to the vial before slide preparation. Good quality Raman spectra were recorded from negative and high grade $(\mathrm{HG})$ cytology samples with no blood contamination and with heavy blood contamination. Good classification between negative and $\mathrm{HG}$ cytology could be achieved for samples with no blood contamination (sensitivity $92 \%$, specificity $93 \%$ ) and heavy blood contamination (sensitivity $89 \%$, specificity $88 \%$ ) with poorer classification when samples were combined (sensitivity $82 \%$, specificity $87 \%$ ). This study demonstrates for the first time the improved potential of Raman spectroscopy for analysis of ThinPrep specimens regardless of blood contamination. $\odot 2018$ Society of Photo-Optical Instrumentation Engineers (SPIE) [DOI: 10.1117/1.JBO 23.5.0550011
\end{abstract}

Keywords: Raman spectroscopy; cytology; ThinPrep; cervical precancer; cervical cancer; blood; blood removal. Paper 170815R received Dec. 19, 2017; accepted for publication Apr. 16, 2018; published online May 4, 2018.

\section{Introduction}

Cervical cancer is the fourth most common cancer in women worldwide with an estimated 528,000 cases and 266,000 deaths in 2012. ${ }^{1}$ The link between cervical cancer and high risk human papilloma virus (HPV) infection and the molecular control of the cell cycle is firmly established. ${ }^{2}$ The presence of an HPV infection does not automatically mean the development of cervical cancer or precancer [cervical intraepithelial neoplasia (CIN)]. However, the HPV virus can integrate its viral DNA into the host cell's DNA located in the cell's nucleus. This integration disrupts the host cell's control of cell growth and replication and can lead to malignant transformation. ${ }^{3}$ The most common method for cervical screening is based on the papanicolaou test (pap test), which is designed to screen for signs of precancer and cancer of the cervix. The pap test requires cells to be scraped from the cervix, fixed to a glass slide, stained, and reviewed by a trained cytologist. Cellular abnormalities are identified based on cellular morphology and staining characteristics and classified according to the degree of dysplasia. The ThinPrep liquid-based cytology (LBC) method involves the patient's cells being transferred into a specimen vial containing PreservCyt transport medium. PreservCyt is an alcohol-based solution that preserves cell morphology and breaks down biological components, such as mucin and blood. The normal

*Address all correspondence to: Damien Traynor, E-mail: Damien.traynor@ dit smear pattern is made up of epithelial and nonepithelial components [Fig. 1(a)]. The epithelial cell components include parabasal cells, intermediate cells, superficial cells, endocervical and endometrial cells. Nonepithelial cell components include red blood cells, lymphocytes, histiocytes, polymorphs, mucus, and bacteria. Red blood cells are 7 to $8 \mu \mathrm{m}$ in size and stain red or pink with the pap stain. They are often found in cervical samples, either due to menstruation or inflammation and their presence can also be used as an indicator of disease (precancer). ${ }^{4}$ A patient's cervix with a high-grade (HG) lesion will often bleed on contact with the cervical brush and, as a result, the smear becomes contaminated with blood. The presence of blood in the sample will often dilute diagnostic cells, cover and surround cell groups, increase screening time, and make microscopic interpretation difficult [Fig. 1(b)]. PreservCyt contains a lysis agent for mucus and red blood cells but excessive blood in the sample vial will prevent a satisfactory smear being produced. High volumes of red blood cells will prevent the collection of epithelial cells onto the polycarbonate filter membrane during processing resulting in a ringed halo effect with red blood cells deposited mostly around the edges of the smear with very few epithelial cells present [Fig. 1(c)].

Cytology laboratories often annotate pap smears according to a blood scale index, where 0 indicates no visible evidence of blood present and 3 indicates an extremely bloody sample (Fig. 2). Samples presenting as a 2 or 3 on the blood scale are

$1083-3668 / 2018 / \$ 25.00$ @ 2018 SPIE 


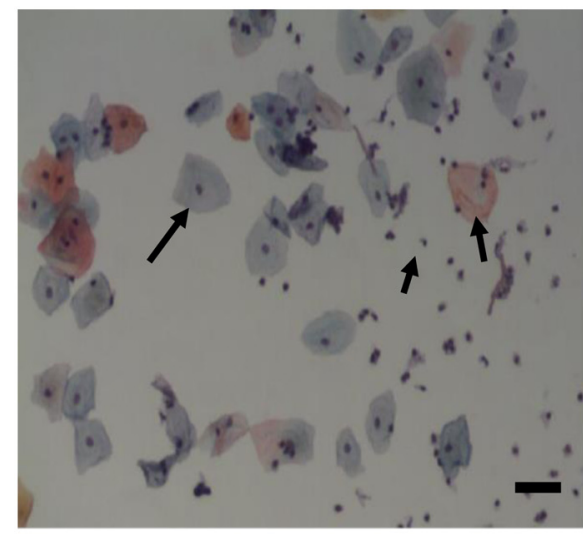

(a)

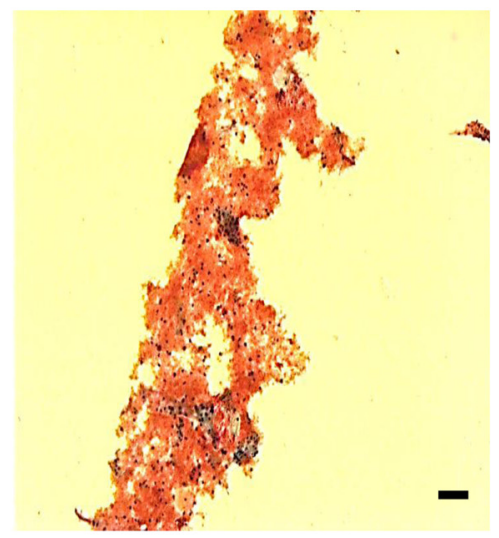

(b)

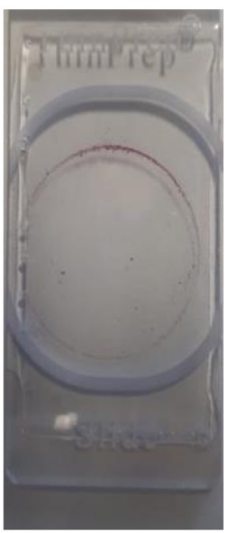

(c)

Fig. 1 (a) Pap smear after staining. Note the presence of intermediate (blue), superficial (pink) cells, and lymphocytes (indicated by arrows), (b) bloody smear pattern with red blood cells, obscuring diagnostic cells. Bar $=35 \mu \mathrm{m}$. (c) Pap smear contaminated with blood showing ringed halo effect.

often either rejected as being unsatisfactory or treated with a wash solution to lyse more of the red blood cells to increase the number of epithelial cells present on the slide. This blood scale grading is subjective and is only based on a visual appearance of a sample. The advantage of the pap test is that it is a noninvasive and widely accepted screening-based test. However, despite a high specificity of $96.5 \%$, a lower sensitivity of $85 \%$ can be due to sampling, technical and interobserver errors associated with the subjectivity of the test. ${ }^{4}$ Over the past 20 years, Raman spectroscopy has shown potential for the diagnosis of cervical cancer both in vivo and ex vivo. ${ }^{5-12}$ Raman spectroscopy is based on inelastic light scattering in which a sample is illuminated by monochromatic laser light and interactions between the incident photons and molecules in the sample result in the scattering of the light. The coupling of the light generates vibrations within the sample, which are characteristic of the chemical structure. The energy of the scattered light is reduced by an amount equal to the vibrational energy, which is called the Raman shift. This means that the position, peaks, and shape of the Raman bands carry information about the molecular makeup of the sample. The Raman spectrum of cells and tissues is made up of contributions from many biochemical components, including DNA, RNA, proteins, lipids, and carbohydrates. ${ }^{12}$ To date, most likely due to confounding factors, such as blood contamination, there have been relatively few studies

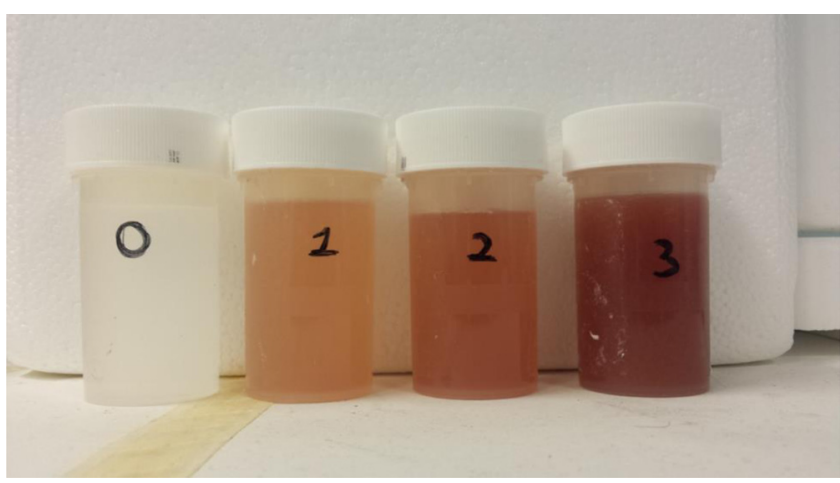

Fig. 2 ThinPrep vials graded according to the blood scale index, where 0 indicates no visible evidence of blood and 3 indicates an extremely bloody sample. on Raman spectroscopy of cervical smear samples. ${ }^{13-19}$ Rubina et al. ${ }^{16}$ described the influence of blood on the Raman spectra of cervical cell pellets and how this might lead to false interpretations. Classification efficiency of $86 \%$ and $84 \%$ was reported for normal and abnormal samples, respectively, and this decreased to $78 \%$ and $79 \%$ after samples were treated with red blood cell lysis buffer to reduce blood contamination. A previous study by our group ${ }^{17}$ addressed many of the issues involved in recording Raman spectra from LBC (ThinPrep) samples and described a method to remove blood contamination before Raman spectroscopy based on pretreatment of the slides with hydrogen peroxide. This was shown to minimize variability and to result in the collection of highly reproducible data with excellent discrimination between negative and HG samples. However, only blood scale 0 and blood scale 1 samples were used for this study as the treatment was not found to be effective for excessively contaminated blood scale 2 and 3 samples. Kerr et al. ${ }^{20}$ investigated the effect of blood contamination on Raman spectra of urine cytology samples. They determined that spectra recorded from samples with a low level of blood appeared free from contamination due to the effective removal of many of the red blood cells using the lysing agent CytoLyt. However, for excessively bloody samples, several additional Raman peaks associated with blood were observed leading to the conclusion that excessively bloody samples are not suitable for diagnostics with Raman microspectroscopy. The aim of the present study was to extend our previous study ${ }^{17}$ to samples annotated as blood scale 2 and 3 to investigate if these excessively bloody samples could be treated to render them suitable for Raman spectroscopy.

\section{Materials and Methods}

\subsection{Samples and Blood Treatment}

Cervical LBC samples were provided by Coombe Women and Infants University Hospital, Dublin, Ireland, for spectroscopic analysis with the final clinical report prepared by the cytologist and/or pathologist. This study was approved by the Research Ethics Committee at Coombe Women and Infants University Hospital. A set of 30 samples were selected, 15 HG intraepithelial neoplasia samples, which received a CIN 2 or CIN 3, result on their associated biopsy from pathology, and 15 negative samples $(\mathrm{TN})$, which received a negative result from cytology. All 
cytology samples were evaluated by eye according to the blood scale index and annotated as being either a grade $0,1,2$, or 3 .

HeLa cervical cancer cells were also prepared and fixed as for ThinPrep samples in PreservCyt. These samples were seeded with blood to mimic excessively bloody cytology samples. This was achieved by adding blood to the samples until they visually represented a blood scale 2 and 3 index sample.

Each ThinPrep sample vial was mixed for 3 min to break up cellular clumps. The sample was then poured into a 50-ml centrifuge tube and centrifuged for $5 \mathrm{~min}$ at $300 \mathrm{rpm}$. The supernatant was removed and $1 \mathrm{ml}$ of $\mathrm{H}_{2} \mathrm{O}_{2}$ was added and mixed for $30 \mathrm{~s}$. About $20 \mathrm{ml}$ of PreservCyt was added to neutralize the $\mathrm{H}_{2} \mathrm{O}_{2}$. The sample was then centrifuged for $5 \mathrm{~min}$ at $600 \mathrm{rpm}$. Most of the supernatant was removed. The cell pellet was resuspended multiple times with a $1-\mathrm{ml}$ pipette to break up cell clumps. The $20 \mathrm{ml}$ of PreservCyt was then added and the contents poured back into the original vial.

\subsection{ThinPrep Slide Preparation}

The samples were then prepared using the ThinPrep 2000 processor (Hologic Inc., Marlborough, Massachusetts). The ThinPrep process begins with the patient's gynecological sample being collected by the clinician using either a cervical broom or brush. The brush/broom is then rinsed in the specimen vial containing PreservCyt transport medium (ThinPrep Pap Test; Cytyc Corporation, Boxborough, Massachusetts). The ThinPrep sample vial is then capped, labeled, and sent to the lab to be processed. The ThinPrep processor homogenizes the sample by spinning the filter and creating shear forces that breaks up any clumped material (blood, mucin, and nondiagnostic material). The cells are then transferred onto a polycarbonate filter membrane of the TransCyt filter and transferred onto a glass slide to produce a circular monolayer of cells $\sim 20 \mathrm{~mm}$ in diameter. The slide is then ejected into a fixative bath of $95 \%$ ethanol.

\subsection{Raman Spectroscopy}

All Raman analyses was performed using a HORIBA Jobin Yvon XplorRA $^{\mathrm{TM}}$ system (Villeneuve d'Ascq, France), which incorporates an Olympus microscope BX41 equipped with a $\times 100$ objective (MPlan, Olympus, NA $=0.9$ ). A 532-nm diode laser source was used. Laser power was set to $100 \%$ resulting in $16 \mathrm{~mW}$ at the objective. The confocal hole coupled to a slit aperture of $100 \mu \mathrm{m}$ was set at $100 \mu \mathrm{m}$ for all the measurements. The system was precalibrated to the $520.7 \mathrm{~cm}^{-1}$ spectral line for silicon. About 1200 lines/grating mm were used. The backscatter light was measured using an air-cooled $\mathrm{CCD}$ detector (Andor, $1024 \times 256$ pixels). The spectrometer was controlled by Labspec V5.0 software. Two accumulations of $30 \mathrm{~s}$ were performed on each cell nucleus selected. Raman spectra were acquired from the nuclei of randomly selected morphologically normal superficial and intermediate cells from each unstained pap smear. (a)

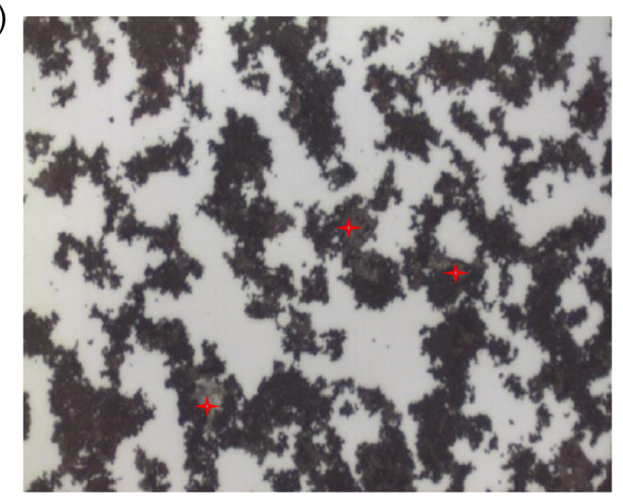

(c)

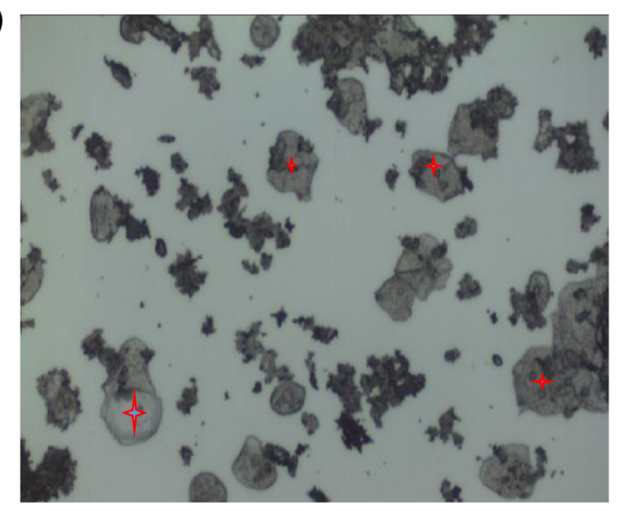

(b) $7 \times 10^{4}$

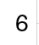

5

4

3

2

1

$\begin{array}{lllcrrr}0 & 600 & 800 & \begin{array}{c}1000 \\ \text { Wavenumbers }\left(\mathrm{cm}^{-1}\right)\end{array} & 1400 & 1600 & 1800\end{array}$

(d)

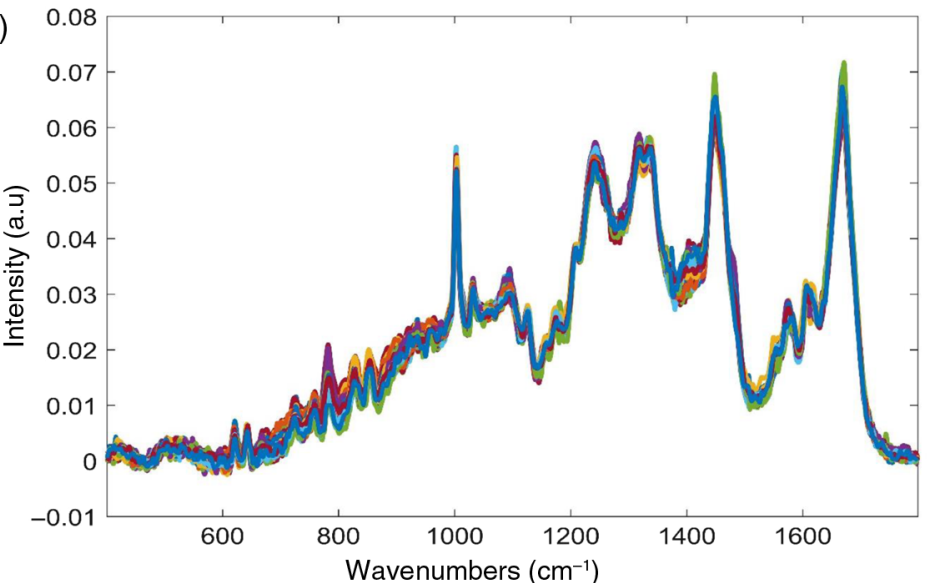

Fig. 3 (a) Untreated pap smear, blood scale 3, stars indicate recorded nuclei in field-of-view. Bar $=35 \mu \mathrm{m}$. (b) Raman spectra recorded from untreated pap smear. (c) Treated pap smear, stars indicate recorded nuclei in field-of-view. Bar $=35 \mu \mathrm{m}$. (d) Raman spectra recorded from treated pap smear. 

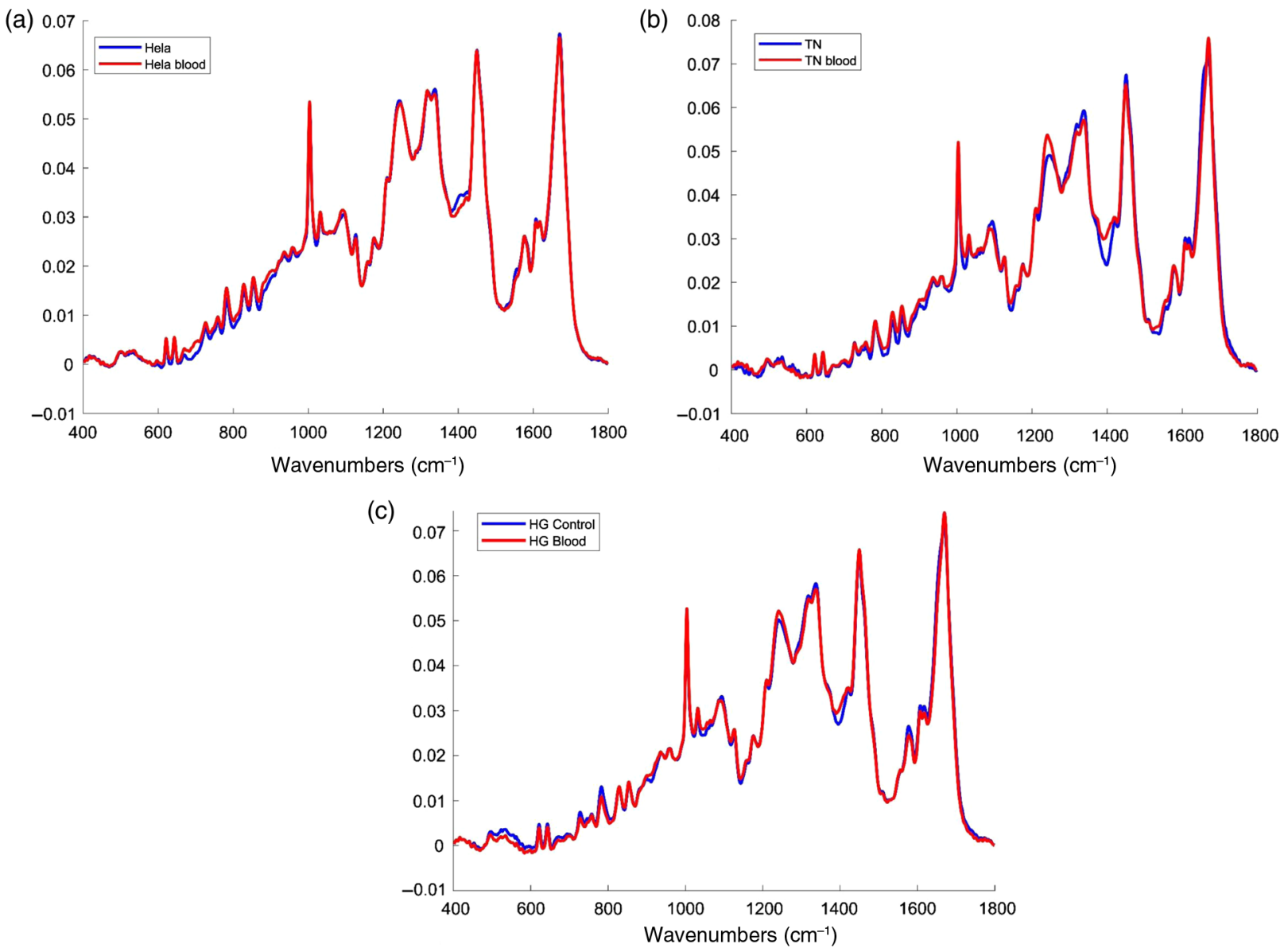

Fig. 4 (a) Mean Raman spectra of HeLa cervical cancer cells treated with blood (blood scale 2 and 3) and subsequently treated with $\mathrm{H}_{2} \mathrm{O}_{2}$ (red) and HeLa cervical cancer cells not treated with blood but treated with $\mathrm{H}_{2} \mathrm{O}_{2}$ (blue), (b) mean Raman spectra of true negative samples (TN), blood scale 2 and 3 , treated with $\mathrm{H}_{2} \mathrm{O}_{2}$ (red) and blood scale 0 , treated with $\mathrm{H}_{2} \mathrm{O}_{2}$, (c) mean Raman spectra of HG samples, blood scale 2 and 3 treated with $\mathrm{H}_{2} \mathrm{O}_{2}$ (red) and blood scale 0, treated with $\mathrm{H}_{2} \mathrm{O}_{2}$.

\subsection{Data Preprocessing and Analysis}

Data were normalized and analyzed using MATLAB software (Mathworks) and specific scripts developed and adapted for uploading of the spectra and their preprocessing, including smoothing (Savitzky-Golay $K=5, K=13$ ) baseline correction (rubberband) and vector normalization. The spectra were also corrected for the glass background using a linear leastsquares method with non-negative constraints. The least-squares model was developed using spectra from the ThinPrep glass slides and selected pure biochemicals (e.g., actin, collagen, RNA, DNA, etc.) that approximate the biochemical composition of cervical cells. The data were mean centered and subjected to partial least squares discriminant analysis (PLS-DA). PLS-DA involves the creation of latent variables to maximize the covariation between known datasets and the response variable which they are regressed against. PLS-DA is a form of analysis that has the ability to distinguish between known classifications of samples (negative, $\mathrm{HG}$ ) and its aim is to find latent variables and directions to maximize separation in a multivariate space. To validate the method leave one patient out cross validation was performed. This involves data from one patient sample being removed from the model and the process is repeated until all samples have been left out once.

\section{Results}

Figure 3(a) shows an untreated Pap smear, blood scale 3. The slide was coated in blood and cellular debris. Figure 3(b) shows Raman spectra recorded from cells present on the slide. Very few cells were present and often burned on contact with the laser. A high background was observed together with Raman bands associated with blood at 754, 1311, 1374, 1398, 1588, $1640 \mathrm{~cm}^{-1}$. The high background can be attributed to the cellular debris or a subunit of the oxidized hemoglobin on the surface of the cell nuclei, which has a weak fluorescence. ${ }^{11}$ Figure 3(c) shows the same sample from Fig. 3(a) after undergoing the blood removal method. Any blood residue has been oxidized with the $\mathrm{H}_{2} \mathrm{O}_{2}$ treatment and cellular debris has been reduced allowing for high quality spectra to be recorded as demonstrated in Fig. 3(d).

In order to determine if the Raman specific method of blood removal using $\mathrm{H}_{2} \mathrm{O}_{2}$ had any effect on the spectra recorded without any external confounding factors, such as inflammation, HPV infection, etc., the protocol was performed on HeLa cervical cancer cells. HeLa cells were seeded with blood to mimic cytology samples with blood scale index 2 and 3 and HeLa cells with no added blood acted as controls. Both sample types were treated with $\mathrm{H}_{2} \mathrm{O}_{2}$ and slides prepared as for the ThinPrep 
cytology samples. Figure 4(a) shows the mean Raman spectra recorded from $30 \mathrm{HeLa}$ cells with and without blood contamination (blood scale 2 and 3) and treated with $\mathrm{H}_{2} \mathrm{O}_{2}$. The mean spectra display strong similarities with only minor changes at $1242 \mathrm{~cm}^{-1}$ (Amide III), $1350 \mathrm{~cm}^{-1}$ (DNA/RNA, CH def. in proteins and carbohydrates), $1430 \mathrm{~cm}^{-1}$ (protein, lipids), and $1669 \mathrm{~cm}^{-1}$ (Amide I). Figure 4(b) shows the mean Raman spectra of negative cytology samples (TN) with and without blood contamination (blood scale 2 and 3) and treated with $\mathrm{H}_{2} \mathrm{O}_{2}$. The mean spectra display strong similarities with small changes at $1242,1350,1430$, and $1669 \mathrm{~cm}^{-1}$ as observed for the HeLa cells. These small changes in the spectra were consistent throughout all samples tested including the HG samples [Fig. 4(c)].

PLS-DA classification of negative cytology samples and HG cytology samples, blood scale index 0 , is shown in Fig. 5. Figure 5(a) is a latent variables (LV) scores scatter plot of LV1 and LV2, which shows good discrimination along LV1. The loadings, as shown in Fig. 5(b), show that the discrimination is based around Raman peaks at 482, 851, 936, 1128, 1338, $1449,1572,1655$, and $1669 \mathrm{~cm}^{-1}$, which are mainly related to DNA, proteins, and glycogen. Figure 5(c) is a PLS-DA prediction plot showing good classification of negative and HG samples with a sensitivity of $92 \%$ and a specificity of $93 \%$ (Table 1).

PLS-DA classification of TN and HG cytology samples, blood scale index 2 and 3, is shown in Fig. 6. Both negative and HG samples have undergone the Raman-specific blood removal method. Figure 6(a) is a LV scores scatter plot of treated TN and HG samples, which show overlap between the samples. The LV1 loadings, as shown in Fig. 6(b), show that the discrimination is based around Raman peaks at 482, 549, 851, 936, $1145,1182,1237,1338,1449,1576$, and $1669 \mathrm{~cm}^{-1}$ and are similar to the loadings shown in Fig. 5(b). The PLS-DA prediction plot is shown in Fig. 6(c) and has a sensitivity of $89 \%$ and a specificity of $88 \%$ (Table 1 ).

In order to determine if excessively bloody samples could be treated the same way as nonbloody samples in terms of data analysis, the Raman spectral data from the two groups (blood scale 0 and blood scale 2 and 3) were combined. Figure 7(a) shows the LV scores scatter plot, which highlights decreased discrimination between TN and HG cytology. However, the loadings shown in Fig. 7(b) remain similar to the loadings shown in Figs. 5(b) and 6(b) with Raman peaks at 482, 851, $1129,1184,1251,1338,1449$, and $1669 \mathrm{~cm}^{-1}$. The PLS-DA prediction plot is shown in Fig. 7(c) and has a sensitivity of $82 \%$ and a specificity of $87 \%$ (Table 1 ).

Interestingly, the loadings $(482,851,936,1149,1338$ and $1669 \mathrm{~cm}^{-1}$ ) remain almost the same between all three datasets, blood scale 0 , blood scale 2 and 3 and combined, confirming that glycogen, DNA, and proteins are the main discriminating features between negative cytology and HG cytology samples regardless of blood contamination. Similar findings have
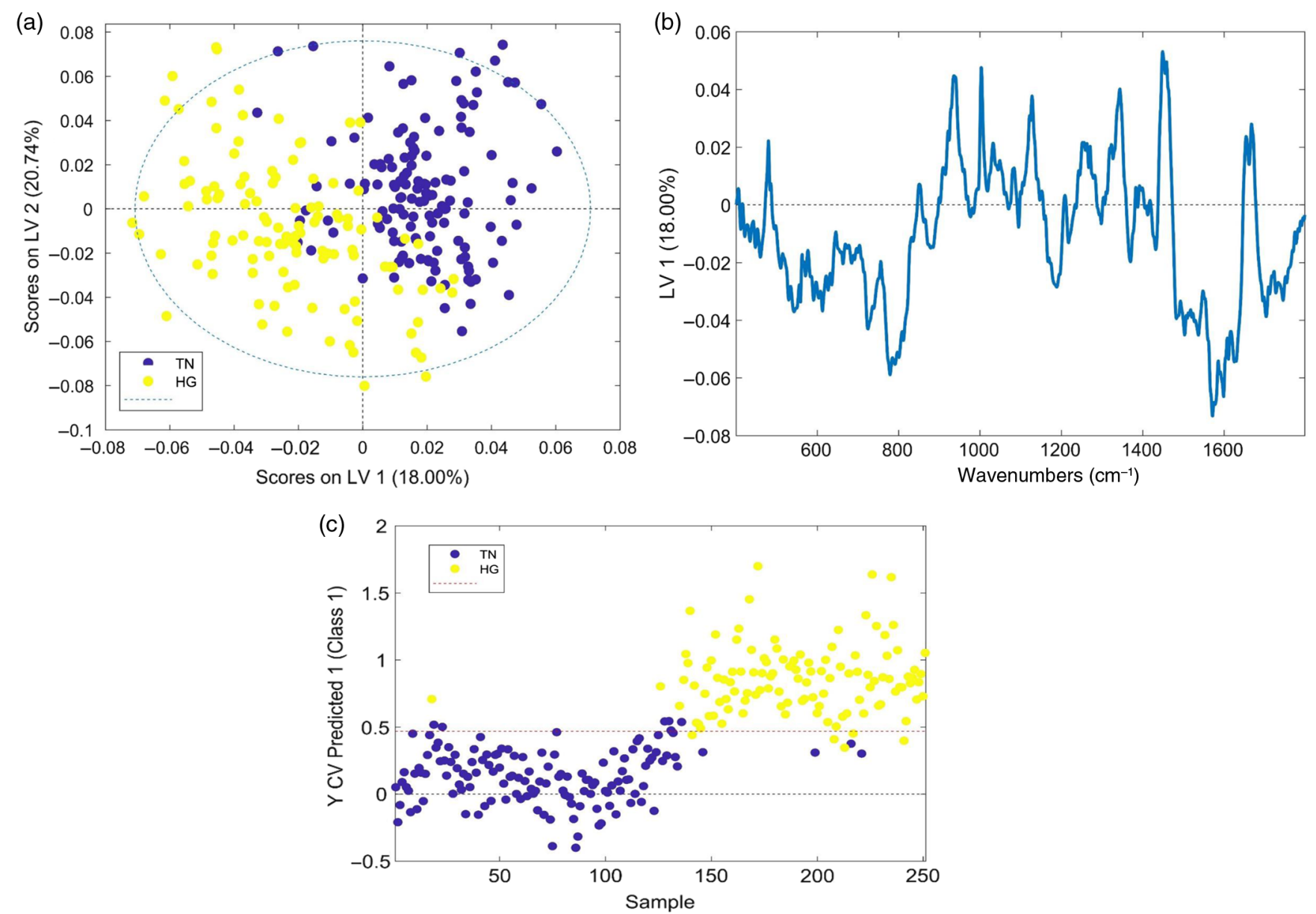

Fig. 5 (a) LV score scatter plot of Raman spectra acquired from 15 TN samples and 15 HG samples (blood scale index 0), (b) LV 1 loadings, and (c) PLS-DA prediction plot showing good discrimination between TN (blue) and HG (red) ThinPrep cytology samples. 
Table 1 Sensitivity and specificity from PLS-DA classification of TN and HG cervical cytology samples with and without blood contamination and combined.

\begin{tabular}{lcc} 
& Sensitivity (\%) & Specificity (\%) \\
\hline $\begin{array}{l}\text { Negative versus HG } \\
\text { (blood scale 0) }\end{array}$ & 92 & 93 \\
$\begin{array}{l}\text { Negative versus HG } \\
\text { (blood scale 2 and 3) }\end{array}$ & 89 & 88 \\
$\begin{array}{l}\text { Combined (blood scale 0 } \\
\text { and 2 and 3) }\end{array}$ & 82 & 87 \\
\hline
\end{tabular}

been reported previously. ${ }^{17,19}$ However, as mentioned earlier, some spectral features undergo minor changes following the blood removal method. As these features overlap with the discriminating features, this results in a reduction in classification efficiency when excessively bloody (blood scale index 2 and 3) samples and nonbloody samples (blood scale index 0) samples are combined for analysis. Comparing sensitivity and specificity of the test groups shows that blood scale 0 samples with no blood contamination performed best with a sensitivity of $92 \%$ and specificity of $93 \%$ when compared to excessively bloody blood scale 2 and 3 samples, which had a sensitivity of $89 \%$ and specificity of $88 \%$. Combining the blood scale 0 and blood scale 2 and 3 samples for analysis resulted in a sensitivity of $82 \%$ and specificity of $87 \%$. The high specificity indicates a good ability to accurately exclude negative samples (few false positives), however, the reduction in sensitivity indicates a reduced ability to accurately detect HG samples and an increased likelihood of false negatives.

Despite the reduction in classification efficiency, the values are higher than previously reported for samples with blood contamination. Rubina et al. ${ }^{16}$ reported a classification efficiency of $78 \%$ and $79 \%$ for normal and abnormal bloody samples treated with red blood cell lysis buffer. In addition, Kerr et al. ${ }^{20}$ concluded that excessively bloody samples were not suitable for diagnostics with Raman spectroscopy.

\section{Conclusion}

This study demonstrates for the first time that excessively bloody (blood scale 2 and 3) ThinPrep cervical smear samples can be used for diagnostics with Raman spectroscopy following a hydrogen peroxide treatment in the collection vial. However, the presence of excessive blood contamination (blood scale index 2 and 3) did result in reduced sensitivity and specificity for classification of negative and HG samples. A higher sensitivity and specificity could be achieved by keeping excessively bloody samples separate from nonbloody samples for analysis. Although this study has focused only on cervical cytology samples, thyroid, urine, and serous fluid cytology samples may also
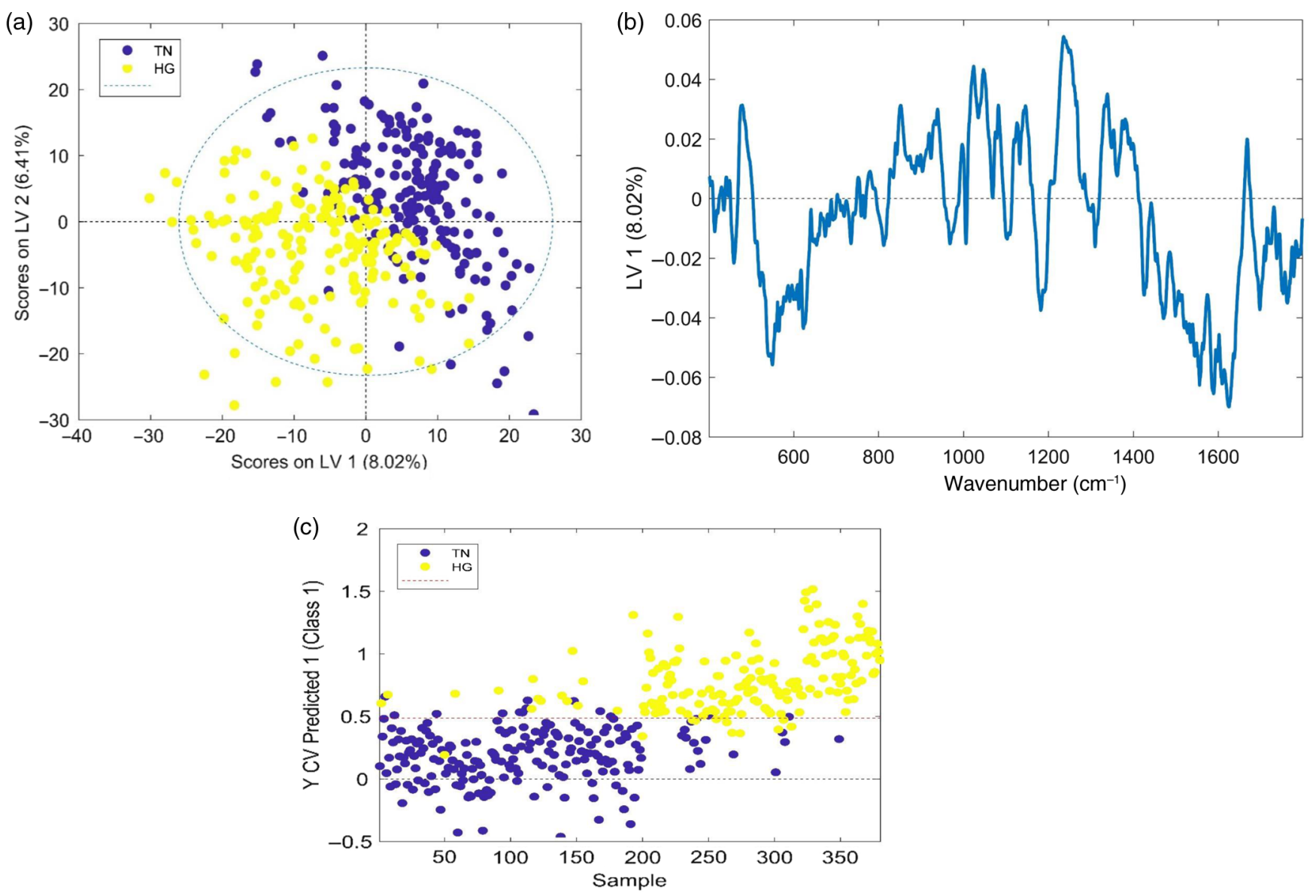

Fig. 6 (a) LV score scatter plot of Raman spectra acquired from 15 TN and $15 \mathrm{HG}$ samples (blood scale index 2 and 3), (b) LV1 loadings, (c) PLS-DA prediction plot showing some discrimination between TN (blue) and $\mathrm{HG}$ (red) ThinPrep cytology samples, blood scale 2 and 3 and treated with $\mathrm{H}_{2} \mathrm{O}_{2}$. 

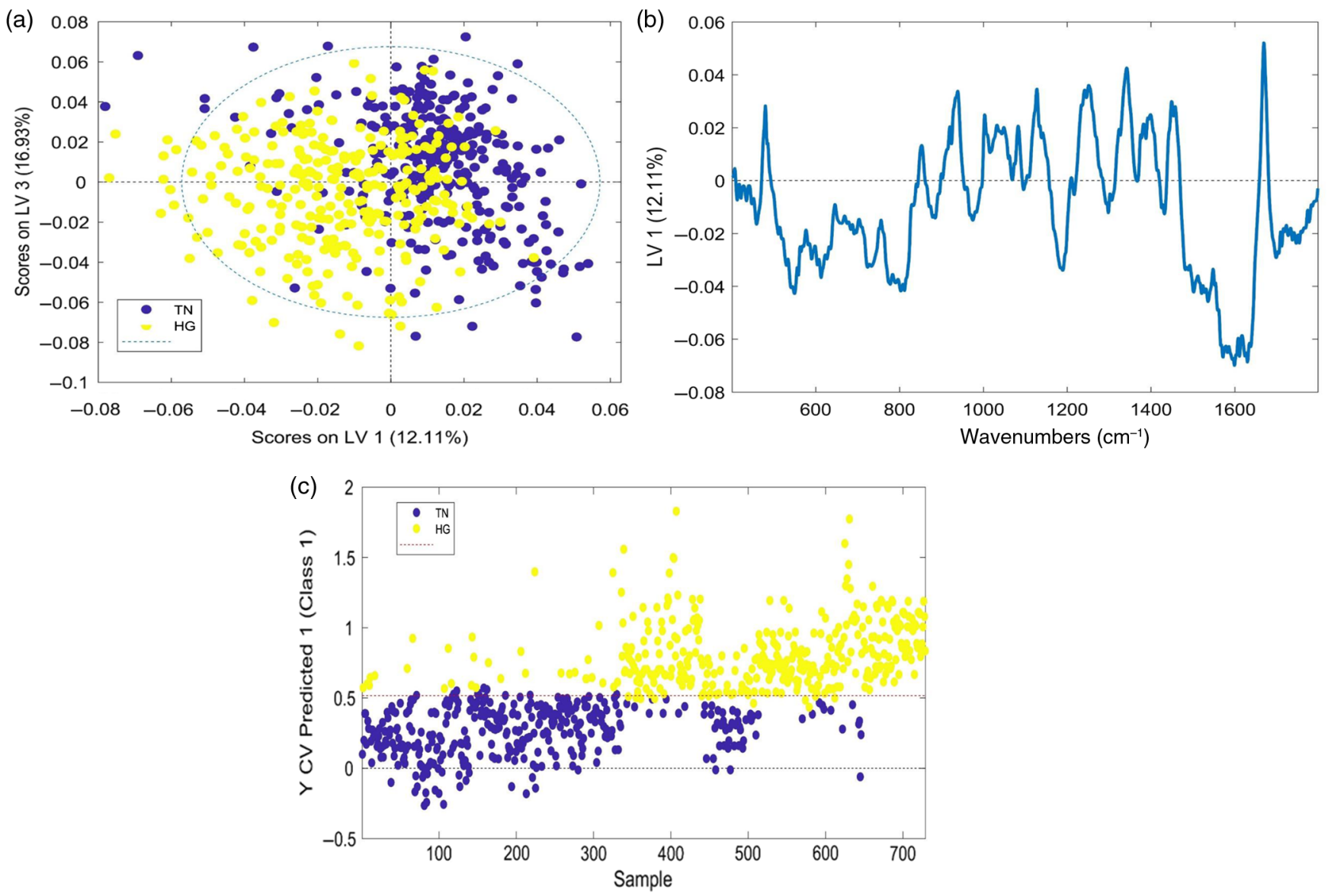

Fig. 7 (a) LV score scatter plot of Raman spectra from TN and HG samples (combined blood scale 0 and blood scale 2 and 3), (b) LV1 loadings, and (c) PLS-DA prediction plot showing some discrimination between TN (blue) and HG (red) ThinPrep cytology samples, combined blood scale 0 and blood scale 2 and 3 and treated with $\mathrm{H}_{2} \mathrm{O}_{2}$.

benefit from such an approach for Raman spectroscopic analysis. Serous fluids for cytological examination are often contaminated with a high level of blood (pericardium, pleura, and peritoneum fluids) and often require additional treatment methods, such a density gradient technique to remove red blood cells, which can result in a loss of diagnostic cellular material. The application of our blood treatment method would prevent this loss of material and allow Raman spectroscopy to be performed.

While these results are promising for the development of Raman spectroscopy for cervical cancer screening, Raman spectroscopy in its current form does not have the ability to replace cytology for cervical cancer screening and its associated benefits (e.g., detection of Herpes Simplex virus or actinomyces like organisms). However, it could be used to supplement the pap test or it could have potential as a triage test after HPV testing.

\section{Disclosures}

There are no conflicts of interest.

\section{Acknowledgments}

This research was undertaken as part of CERVIVA, the Irish Cervical Screening Research Consortium and we gratefully acknowledge funding from the Health Research Board Collaborative Applied Research Grant, CARG2012/29, and Enterprise Ireland cofunded by the European Regional Development
Fund (ERDF) and Ireland's EU Structural Funds Programme 2007-2013, CF2011 1045. We thank the Cytology staff at Coombe Women and Infants University Hospital, Dublin, for facilitating the study and technical support staff at the FOCAS Research Institute, Dublin Institute of Technology.

\section{References}

1. J. Ferlay et al., "Cancer incidence and mortality worldwide: sources, methods and major patterns in GLOBOCAN 2012," Int. J. Cancer 136, E359-E386 (2015).

2. F. X. Bosch et al., "The causal relationship between human papillomavirus and cervical cancer," J. Clin. Pathol. 55, 244-265 (2002).

3. B. Shambayati, Cytopathology, 1st ed., pp. 88-89, Oxford University Press, Oxford (2011).

4. H. C. Kitchener et al., "MAVARIC trial study group," Health Technol. Assess. 15, 1-170 (2011).

5. U. Utzinger et al., "Near-infrared Raman spectroscopy for in vivo detection of cervical precancers," Appl. Spectrosc. 55, 955-959 (2001).

6. E. M. Kanter et al., "Effect of hormonal variation on Raman spectra for cervical disease detection," Am. J. Obstet. Gynecol. 200, 512.e1-512.e5 (2009).

7. E. Vargis et al., "Effect of normal variations on disease classification of Raman spectra from cervical tissue," Analyst 136, 2981-2987 (2011).

8. E. Vargis et al., "Sensitivity of Raman spectroscopy to normal patient variability," J. Biomed. Opt. 16, 117004 (2011).

9. J. Mo et al., "High wavenumber Raman spectroscopy for in vivo detection of cervical dysplasia," Anal. Chem. 81, 8908-8915 (2009). 
10. S. Duraipandian et al., "Simultaneous fingerprint and high-wavenumber confocal Raman spectroscopy enhances early detection of cervical precancer in vivo," Anal. Chem. 84, 5913-5919 (2012).

11. K. Kong et al., "Raman spectroscopy for medical diagnostics-from invitro biofluid assays to in-vivo cancer detection," Adv. Drug Delivery Rev. 89, 121-134 (2015).

12. I. P. Santos et al., "Raman spectroscopy for cancer detection and cancer surgery guidance: translation to the clinics," Analyst 142, 3025-3047 (2017).

13. F. M. Lyng et al., "Raman spectroscopy for screening and diagnosis of cervical cancer," Anal. Bioanal. Chem. 407, 8279-8289 (2015).

14. M. Diem et al., "Molecular pathology via IR and Raman spectral imaging," Biophotonics 6, 855-886 (2013).

15. E. Vargis et al., "Near-infrared Raman microspectroscopy detects highrisk human papillomaviruses," Transl. Oncol. 5, 172-179 (2012).

16. S. Rubina, M. S. Vidyasagar, and C. M. Krishna, "Raman spectroscopic study on prediction of treatment response in cervical cancers," J. Innovative Opt. Health Sci. 6, 1350014 (2013).

17. F. Bonnier et al., "Processing ThinPrep cervical cytology samples for Raman spectroscopy analysis," Anal. Methods 6, 7831-7841 (2014).

18. I. Ramos, A. Malkin, and F. Lyng, "Current advances in the application of Raman spectroscopy for molecular diagnosis of cervical cancer," BioMed Res. Int. 9, 1-9 (2015).

19. P. Kearney et al., "Raman spectral signatures of cervical exfoliated cells from liquid-based cytology samples," J. Biomed. Opt. 22(10), 105008 (2017).

20. L. T. Kerr et al., "Methodologies for bladder cancer detection with Raman based urine cytology," Anal. Methods 8, 4991-5000 (2016).

Damien Traynor holds his BSc degree in medical and molecular cytology from the Dublin Institute of Technology (DIT). He achieved membership of the Academy of Laboratory Science in cytology in 2012 and worked as a primary screener for two years before joining DIT as a research technician. He is working on a Health Research
Board funded clinical evaluation of Raman spectroscopy for the diagnosis of cervical precancer and is working on his PhD part time.

Shiyamala Duraipandian holds her BE degree in biomedical engineering from Adhiyamaan College of Engineering, Anna University. She received her $\mathrm{PhD}$ from the Department of Biomedical Engineering, National University of Singapore. She worked as a Marie-Curie fellow in Dublin Institute of Technology. She is working as a staff scientist in Dansk Fundamental Metrologi A/S. Her research is focused on different analytical techniques for pharm.

Cara M. Martin is an assistant professor in molecular pathology and tumor biology in the Department of Histopathology at the Trinity College Dublin. She leads the Cervical Cancer Research Group based in the Coombe Women and Infant's University Hospital. Her research program consists of translational health services-based research in female gynecological cancers. She has published 57 peer-reviewed articles and 13 book chapters, and has a $h$-index of 23 and $>2500$ citations.

John J. O'Leary is a professor/chair of pathology at the Trinity College Dublin and a director of pathology at the Coombe Women and Infants University Hospital, Dublin. He heads a multi-investigator team focused on the molecular characterization of several cancer systems including: ovary, cervix, prostate, thyroid, head and neck cancer, and cancer stem cell biology. He has published more than 190 peerreviewed papers, and has a $h$-index of 49 and $>9600$ citations.

Fiona M. Lyng holds her BSc degree from Trinity College Dublin and her PhD from the University College Dublin. She is a head of the DIT Centre for Radiation and Environmental Science. Her research is focused on translational research for cancer diagnosis and cancer treatment. She has published over 100 peer-reviewed research papers, and has a $h$-index of 33 and $>3500$ citations. 